\title{
Los nombres de flores y plantas en el diccionario de Cuveiro
}

Carmen González Orejón

Universidade da Coruña

\section{BREVE RESUMEN HISTÓRICO DEL ESTUDIO DE LA BOTÁNICA GALLEGA}

En el siglo XVIII surge el interés por la flora gallega que tiene su figura más destacada en el Padre Fray Martín Sarmiento, un gran estudioso de la cultura gallega en todos los ámbitos, pero con una especial inclinación por las ciencias naturales. Sarmiento redactó su Catálogo de voces vulgares y en especial de voces gallegas de diferentes vegetables entre 1754 y 1755 , inspirado por viajes anteriormente emprendidos a Galicia.

Joseph Quer publica en 1762 Flora española o Historia de las plantas que se crían en España. El primer catedrático de Botánica del Real Jardín de Madrid, animado por el mismo Padre Sarmiento, recorrió durante mucho tiempo gran parte de Galicia recogiendo más de cien plantas diferentes para la publicación del primer tomo de su obra, que cuenta además con laboriosas ilustraciones.

A finales de ese mismo siglo llega a Galicia el sacerdote francés Pourret. Emigrado en la época revolucionaria de su país, recorrió parte de las provincias de A Coruña, Ourense y Lugo elaborando un amplio herbario que sirvió de base para posteriores trabajos de botánica.

En el año 1850 se publica Recuerdos botánicos de Galicia de Miguel Colmeiro y Penide, catedrático de Botánica en la Universidad de Sevilla. En la parte introductoria de este folleto de 22 páginas se hace 
un resumen de los trabajos más valiosos para la botánica, no sólo gallega, sino también española.

El botánico catalán José Planellas Giralt publica en el año 1852 el Ensayo de una flora fanerogámica gallega. Este libro, que incluye nada menos que 853 plantas distintas ofrece una introducción que comienza con un resumen histórico de los trabajos de botánica elaborados en Galicia, continúa con una exposición general de la naturaleza del país y una historia general de la vegetación gallega y finaliza con una relación general de la vegetación gallega comparada con la del resto del mundo y en particular con la de Asturias, León y Portugal. El núcleo del libro lo configura la descripción de 102 familias diferentes de plantas, y concluye con un resumen de los géneros clasificados según el sistema de Linneo.

Para finalizar este breve esbozo de trabajos sobre botánica gallega, cabe mencionar la obra de Baltasar Merino Flora descriptiva e ilustrada de Galicia, publicada en dos tomos en los años 1905 y 1906. El prólogo, lleno de elogios hacia la naturaleza gallega, destaca algunas aportaciones al estudio de la botánica española y gallega, como por ejemplo el Bosquejo histórico de la botánica española de Victor López Seoane, y también las visitas a Galicia de Juan Lange y Mauricio Willkomm, que plasmaron los resultados de las mismas en el Prodomus Florae Hispanicae (1861-80) y en el Supplementum Prodomi Hispanicae (1893). El número de plantas descritas por estos dos autores ascendía a más de 1000 ejemplares, pero la descripción de Merino supera esta cifra considerablemente, ya que, como admite en el prólogo, "reconocemos el auxilio que varios amigos nos han prestado, preparando, enviándonos o poniendo a nuestra disposición sus colecciones de plantas" (1905:VI).

Podemos observar la abundancia de obras, unas más precisas y extensas que otras, pero todas con la finalidad de aportar lo más posible al conocimiento de la botánica gallega. Este despertar del sentimiento de lo autóctono había de verse reflejado también en las obras lexicográficas del momento, tal y como ocurre en el diccionario de Juan Cuveiro Piñol. La ambición de la época del Rexurdimento se evidencia en el extenso título de la obra: Diccionario gallego. El más completo en términos y acepciones de todo lo publicado hasta el día con las voces anti- 
guas que figuran en códices, escrituras y documentos antiguos, términos familiares y vulgares y su pronunciación para la escuela de diplomática, anticuarios, jueces, abogados, escribanos, párrocos y otras personas a quienes es indispensable su frecuente uso (1876).

Vamos a comenzar este trabajo centrándonos en primer lugar en el tratamiento general que reciben los nombres de plantas en el diccionario de Cuveiro, intentando agrupar los diferentes artículos según la información que aporten. Seguidamente expondremos las fuentes principales utilizadas por Cuveiro para la elaboración de sus artículos de botánica, con la intención de determinar en qué medida fueron aplicadas. El último apartado versará sobre las equivalencias gallego-castellanas y latinas de los nombres de flores y plantas, conforme a las fuentes empleadas por Cuveiro.

\section{EL TRATAMIENTO LEXICOGRÁFICO DE LOS NOMBRES DE FLORES Y PLANTAS EN EL DICCIONARIO DE CUVEIRO}

Tras un análisis de los artículos referidos a botánica en el diccionario de Cuveiro Piñol, cuyo número asciende a 779 entradas, podemos constatar que son agrupables de acuerdo con sus características, en tres grandes grupos, aunque cabe destacar que el rasgo común que tienen todas estas definiciones es su carácter enciclopédico ${ }^{1}$.

En primer lugar, un grupo de artículos enciclopédicos ofrecen la siguiente información: después de la entrada se incluye, si es el caso, el nombre equivalente en castellano ${ }^{2}$ con la indicación de su pertenencia a una determinada familia o subgénero; a continuación se procede a una detallada información sobre la especie, su lugar de crecimiento y otras características como color, forma de las hojas, flor u olor. A veces figu-

\footnotetext{
1 Recordemos que las definiciones enciclopédicas o "definiciones de predicación múltiple", como las denomina Seco suelen aplicarse sobre todo en aquellos nombres "que designan seres u objetos materiales, y preferentemente en los que corresponden a zoología y botánica" (1987:30/31).
}

2 Haremos hincapié en esta cuestión en el apartado 4. 
ra alguna indicación acerca de la aplicación médica. La denominación latina de cada especie puede aparecer en el interior de la parte definitoria o al final. Veamos algún ejemplo:

Berrofemia - berrera, planta que nace y muere durante el año, frutos aovados, globosos: crece a orillas de los arroyos y en tierras cenagosas: su tallo es estriado, tendido, y sus hojas pinado-cortadas; de la subfamilia recto semilladas, tribu ammíneas. Heloiciadium Suim modiflorum, con su variedad Sison inundatium, de tallo rastrero que crece en el cauce del Sar, junto al molino de la Rocha vieja.

Pleastro - planta anual de la tribu trifolieas, de tallo tendido, pelosa, hojas trasovadas dentelladitas, flores blancas pasando al amarillo verdoso; crece en los lugares estériles de las cercanías de la Coruña, y es común en el glasis de la Puerta de la Torre. Phleastrum scabrum.

Gilbarbeira - mata ó arbusto de la familia asparrágeas, llamada Brusco y Jusbarba, de tallo rígido ramoso, hojas elíptico lanceoladas, punzantes, desnudas y que llevan flor en la faz, comun en las selvas; raíces dulce-amargas aperitivas y diuréticas; estas y los tallos tiernos sirven contra hidropesía, obstrucciones y desgana; sus ramas se usan para desarañadores. Ruscus aculeatus.

Genciana - planta que nace y muere durante el año, de la familia gencianáceas, de hojas anchas, aovadoas, nerviosas, corola en forma de campana o rueda, amarilla: crece en los montes que circuyen el valle de Lemos; sus raíces, de sabor dulce y después muy amargo son aromáticas, altamente tónicas y febrífugas, succedáneas de la quina, Gentiana lutea, con su variedad Pneumonanthe, de hojas lineares lanceoladas, flores terminales o axilares sentadas, corolas azules campanuladas: crece en los prados húmedos.

El segundo grupo lo componen 69 artículos que se limitan simplemente a una remisión. Algunos remiten a la misma planta, herba velleira, avelleira y melisa, que remiten todas a trungil. Asimismo se remite dos veces a gilbarbeira (mesquita, jusbarba), muruxas pajareira, pica galiña) y malvela (herba de reste, herba redondo) ${ }^{3}$.

3 En muchos casos no se indica que se trata de una planta, lo cual dificulta un poco la búsqueda para los no instruídos en este ámbito. 
El tercer grupo está formado por 29 artículos que, aparte de remitir a otros nombres de plantas, constan de una definición enciclopédica que puede ser más o menos extensa. Veamos algún ejemplo:

Infrade - planta que nace y muere en el año, de la familia cariofíleas, tribu siléneas, de tallos postrados, ramosos, hojas lineares, angostadas, en ambos estremos, acanaladitas, pestañosas, recurvadas, crasas, flores terminales, solitarias o casi umbeladas; las hojas son de media a una pulgada de longitud por una línea de latitud, por lo común más largas que los entrenudos, pestañas de sus bordes formadas por dientes cartilaginosos, numerosísimos, pedúnculos erguidos y flores blancas; crece en los terrenos quebrados lindantes con los arenales de la Isla de Tambo y de Combarro. Inflata Stenophylla Angustofolia; con su variedad Miera, de tallos cortos, apretados contra la tierra, casi solitarios, hojas de cuarta a tercia de longitud comparadas con las del tipo; crece en las vertientes marítimas de la Torre de Hércules e inmediatas al castillo de San Diego en la Coruña, y también en varias otras de la ría de Pontevedra. V. Coleja.

Pelitre - V. Peletre - planta de raíz gruesa y larga, tallos sin ramas y terminados en una flor grande y hermosa, compuesta de varias hojas o pétalos blancos por encima y de color de púrpura por el embés, que salen de un centro común, de color amarillo: las hojas se componen de otras recortadas en tiras sumamente delgadas. Anthemis pyrethrum.

Como constatación global en cuanto a las remisiones, podemos señalar que no se indica la frecuencia de uso o la extensión geográfica de las diferentes denominaciones de una misma planta, salvo en el caso de alfaneiro, a la que se nos envía en tres ocasiones a través de fiafeira, filseira y tilseira mientras que la entrada alfaneiro remite, a su vez, a fiafeira:

Fiafeira - (en el E. del País) árbol de la familia jasmíneas, tribu fraxíneas, de ramas apenas borrosas en el ápice, hojas opuestas lanceoladas, enterísimas, rayas negras, blancas, amarillas ó verdosas. V. Alfaneiro

Tilseira - en el S. del país. V. Alfaneiro

Debido a la homogeneidad de la estructura de los artículos de flores y plantas en el diccionario de Cuveiro, que facilita enormemente su clasificación en grupos, nos atrevemos a deducir que la explicación radica 
en la utilización de determinadas fuentes, de lo que vamos a ocuparnos en el siguiente apartado.

\section{FUENTES UTILIZADAS}

Una de las fuentes principales utilizadas para la redacción de los artículos de flores y plantas es el citado Ensayo de una flora fanerogámica gallega del botánico catalán José Planellas Giralt, que el mismo Cuveiro revela al principio del diccionario. Compárense los siguientes extractos, el primero de los cuales pertenece a Cuveiro:

Arearia - planta anual de la tribu alsíneas, de hojas aleznadas á manera de cerdas de tallo muy ramoso, ahorquillado; crece según Lange en las murallas de Lugo. Arenarium tenuifolia, con sus variedades Montana, pubescente, de hojas lanceolado-lineares, pétalos inversoaovados, de color blanco; crece en los lugares estériles de todos los montes; y la Serpillyfolia, de hojas aovadas, agudas sentadas, rugosas, pestañosas, lisas; en las tierras arenosas de los campos de Lugo. Arenaria espergularia.

A. TENUIFOLIA (Linn. Sp. 607) Sin nomb. Vulg.

Hojas aleznadas, á manera de cerda; tallo muy ramos, ahorquillado; sépalos aleznados, estriados, mucho mas largos que los pétalos; cajas de 3 ventallas, apenas mas largas que los cálices [...] Crece s. Lange en las murallas de Lugo.

A. MONTANA. Pubescente, hojas lanceolado-lineares, tallos estériles larguísimos y postrados [...] Crece en los lugares estériles de todos los montes.

A. SERPYLLIFOLIA (Linn.sp. 606) Sin nomb. Vulg.

Hojas aovadas, agudas, sentadas, rugosas, pestañosas, lisas; tallos ramosísimos, sépalos lanceolados, agudos, de 3-nervios, verdes, opacos, casi doble mas largos que la corola; cajas aovadas, que se abren en el ápice por 6 ventallas, de la longitud del cáliz; semillas exactamente en forma de riñon, rugosas. Variable en los tallos mas o menos ramosos y en las hojas y cálices con pocos o muchos nervios y algunas veces viscositos. Crece s. Lange en las tierras arenosas de los campos de Lugo (1863: 131).

También los siguientes ejemplos, escogidos al azar, corroboran que Cuveiro utilizó el Ensayo de Planellas para la elaboración de los artícu- 


\section{los de botánica. Comenzamos nuevamente con el artículo de Cuveiro.}

Ensalada de sapo - planta anual de la familia portuláceas, lampiña, con hojas opuestas, flores axilares, pequeñas, blancas. Monita Frontana, con su variedad Repens, de tallo débil, rastrero, ahorquillado, hojas aovadas sentadas, en los arroyuelos al S. de Bar.

M. FONTANA (Linn. Sp. 129). Cast. Ensalada de sapo.

Lampiña con hojas opuestas, flores axilares, pequeñas, blancas.

a. Repens (Pers. Ench.1.p.111) Tallo débil, rastrero, ahorquillado; hojas aovadas, sentadas. Crece en los arroyuelos poco profundos al s. de Bar etc, etc. (1863: 214).

Lisimaquion - planta que nace y muere durante el año, de la familia onagrarieas, de tallo erguido, redondo, lampiño, hojoso, hojas lanceoladas irregularmente aserrado-dentadas casi sentadas, lampiñitas: crece, según Lange, en los bosques de Lugo. Epilobium montanum, con sus variedades Hirsutum, de tallo muy ramoso, peludo, hojas opuestas y alternas, lanceolado-alargadas, irregularmente aserradas, abrazaderas peludas, con venas también peludas, flores purpúreas: la Vulgaris, de tallo, ramas y hojas pelosas, frutos cubiertos de pelos cortos, en las tierras inundadas de algunos valles templados; la Parviflorum, de raíz fibrosa, tallo sencillo o ramoso, delgado velloso, hojas sentadas, opuestas y alternas, lanceolado-alargadas, aserraditas, agudas, con bozo blanco, flores purpúreas, en las tierras húmedas y sombrías del Ulla; la Tetragonum, de tallo casi lampiño, hojas lanceolado-alargadas, dentelladitas, las inferiores opuestas, flores purpúreas, en las márgenes de los arroyos, y la Obscurum, de hojas opuestas y alternas, lanceoladas, lampiñas aserradas, decurrentes en la base, estigma a manera de clava.

E. MONTANUM (Linn. Sp. 494).

Tallo erguido, redondo, lampiño, hojoso; hojas lanceoladas, irregularmente aserrado-dentadas, casi sentadas, lampiñitas; piececillos florales cortos, pétalos acorazonado-inversos, más largos que el cáliz; estigma 4-partido, cajas delgadas, alargadas. Crece s. Lange en los bosques de Lugo.

E. HIRSUTUM (Linn. Sp. 494. v.a.)

Tallo muy ramoso, peludo; hojas opuestas y alternas, lanceolado-alargadas, irregularmente aserradas, abrazadora, peludas, con venas también peludas; estigmas 4, abiertos, declinados; sépalos con rejoncito, 
casi lampiños; pétalos escotados, grandes, de 1,2 pulgada de largo.

a. Vulgaris (Ser. in D. C. Prod. 3. p. 42)

Tallo, ramas y hojas pelosas; frutos cubiertos de pelos cortos. Crece en las tierras inundadas de algunos valles templados.

E. PARVIFLORUM (Schreb. Engl. Bot. T. 795). E. HIRSUTUM b (Linn. Sp. 498)

Raiz fibrosa, tallo sencillo ó ramoso, delgado, velloso; hojas sentadas, opuestas y alternas, lanceolado-alargadas, aserraditas, agudas, con bozo blando; flores pequeñas, estigmas 4 , abiertos; cajas vellositas ó casi lampiñas. Con flores purpúreas. Crece en las tierras húmedas y sombrías del valle de la Ulla.

E. TETRAGONUM. (Linn. Sp. 494)

Tallo con 4 ángulos obtusos, casi lampiño; hojas lanceolado-alargadas, dentelladitas, las inferiores opuestas: estigma sencillo. Con flores purpúreas. Crece en las márgenes de los arroyos.

a. Obscurum (Pers. Ench. 1.p. 410) Hojas opuestas y alternas, lanceoladas, lampiñas, aserradas, decurrentes en la base; estigma a manera de clava (1863: 207-208).

Podemos observar que los artículos que ofrece el Ensayo de Planellas, sobre todo los menos extensos, casi se copian en su totalidad. A veces se suele omitir alguna información en la que, por lo general, se trata de detalles muy técnicos, como demuestra el siguiente artículo de la planta echinaria:

Echinária - planta anual de la familia gramíneas, de espiga compuesta en cabezuela; crece en la comarca del Ferrol. Echinaria capitata.

ECHINARIA. Espiga compuesta, en cabezuela. Gluma de 2 brácteas arrejonadas, con 2-4 flores. Glumela de 2 brácteas, la esterior con 5 cerdas lanceoladas, la interior más pequeña, escotada y con 2 cerdas.

E. CAPITATA. Crece en la comarca del Ferrol (1863: 406).

Así pues, la comparación de los artículos refleja con claridad que la fuente bibliográfica principal de Cuveiro en sus artículos de botánica fue el Ensayo de José Planellas Giralt; sin embargo existe otra, el diccionario de Francisco Javier Rodríguez (1863), que es la única fuente que se revela explícitamente en la parte definitoria con la anotación “según Rodríguez". Las nombres que provienen de este volumen son, en su mayoría, denominaciones compuestas, como herba da becerra, 
herba das desentérias, etc., es decir nombres vulgares, que no se acompañan de etimología latina, mientras que otros sintagmas semejantes sí incluyen la denominación latina, (así, por ejemplo, herba dos candiles (arum armisarum), herba dos canónigos (valerianella olitoria) o herba estoque (gladiolus comunis) cuando han sido redactados aprovechando los materiales. Comparemos nuevamente algún artículo:

Herba da sarna - según Rodríguez es la adelfa, planta que se parece algo al laurel, de flores imitantes a las rosas, las que, y sus hojas, son venenosas y contra la sarna [Cuveiro].

Erba da sarna. Adelfa, planta que se parece algo al laurel, cuyas hojas y flores son venenosas y contra la sarna. Da rosas bastante agradables a la vista y por eso el francés la llama laurier-rose y el italiano rosa a secas. En castellano también la llaman rosa da mascena, oleandro, baladre, etc. En port. Id. [Rodríguez].

Herba benta - según Rodríguez, es la valeriana [Cuveiro].

Erba benta. Valeriana [Rodríguez].

En alguno de estos artículos del diccionario de Cuveiro podemos encontrarnos con comentarios por parte del autor en los que, generalmente, manifiesta su desacuerdo con Rodríguez o lo rectifica. Así ocurre, por ejemplo, en la entrada cabrinfollo:

...Rodríguez al describirla con el nombre de Herba salgueira, tal vez se equivoca, pues el convólvulus es la corregüela y no dice su descripción con esta última que verá el lector en su lugar, adviertiendo que no hay otro convolvulus [Cuveiro].

Hallamos otro comentario en el artículo camariñas:

Camariñas - V. Caramiñeira, en lugar del arbusto que describe Rodríguez con el nombre de Camariñas, que no es tal, sino el caramiñeiro [Cuveiro].

Podemos, pues, observar actitudes diferentes de Cuveiro frenta a las fuentes consultadas; los artículos que provienen del Ensayo de Planellas Giralt no se cuestionan ni se comentan (ni siquiera se revela su fuente), cosa que sí se hace en los artículos de botánica basados en el diccionario de Rodríguez. 


\section{LAS EQUIVALENCIAS GALLEGO-CASTELLANAS Y LATINAS}

Como ya señalábamos en el apartado segundo, no siempre se indica en la parte definitoria la correspondencia en castellano del nombre de la flor o planta gallega. La fuente principal de información sobre botánica, el Ensayo de Planellas, ofrecía después de la denominación latina de cada planta la información acerca de los nombres vulgares, en castellano o en gallego, aunque con frecuencia nos encontrábamos con anotaciones, como "sin nombre vulgar en gallego" o "sin nombres vulgares"; sin embargo, los nombres de las respectivas plantas se encuentran incluidos en castellano en el diccionario de Cuveiro. Cuando, según Planellas, no existe nombre vulgar en gallego, Cuveiro incluye la denominación castellana, como advierte en el prólogo:

Por último, también notará el lector algunos términos castellanos, especialmente en plantas ó flores; pero no debe extrañarle esto, atendido el motivo que a ello nos impele, que es dar a conocer el mayor número posible de objetos y producciones cuando estos tienen aplicación a nuestro suelo por sus propiedades médicas, por su hermosura, utilidad, etc., etc., las comarcas o puntos donde mas abundan, o porque los vocablos se pronuncian muy a menudo del mismo modo que en castellano, aunque con acento puramente gallego y que, andando el tiempo, podrá tal vez descubrirse por su etimología, u otra causa, el verdadero nombre que tengan en Galicia, como sucede con infinitas voces de que, a no dudar, ni siquiera tendría la más remota idea de su existencia el lector, de quien reclamamos la mayor indulgencia en gracia del objeto a que aspiramos, cual es el de contribuir con nuestro pequeño óbolo al mayor grado posible de civilización y cultura de tan bello e inolvidable país (1876: 4).

Aquí se plantea la duda si en la ausencia de una equivalencia castellana en la parte definitoria va implícito que la entrada misma ya es el nombre castellano. Para eso hemos consultado el Catálogo da flora vascular galega, de Niño Ricoi, Losada Cortiñas y Castro González (1994), y la Guía da flora do litoral galego, de Luís Alberto Sanmartín y Helena Lago Canzobre (1998), donde hemos comprobado, por ejemplo, que la gentiana lutea, que Cuveiro denomina genciana, sin indicación dentro de la parte definitoria de una correspondencia en castellano, tiene el nombre gallego de xanzá (Niño Ricoi:1994), mientras que 
en Sanmartín (1998) se indica además el nombre castellano genciana de turbera. La lupinus angustifolius, que Cuveiro incluye como altramuz está representada en Niño Ricoi (1994) con el nombre gallego de chícharo de raposo $o^{4}$ mientras que Sanmartín y Lago (1998) sólo ofrecen la denominación castellana altramuz. Vemos que en estos casos se cumple lo anunciado en el prólogo, como se comprueba fácilmente a través de la consulta de las obras de botánica mencionadas, aunque también se nos revelan ciertas incoherencias en la información que proporcionan tanto Cuveiro como los botánicos actuales cuyo trabajo hemos examinado; así, por ejemplo, la potamogeton natans recibe en el diccionario de Cuveiro el nombre de espiga d'ouro, igual que en Niño Ricoi (1994), mientras que carece de nombre gallego en Sanmartín y Lago (1998), y la planta antennaria dioica está representada en el diccionario con la entrada de antenaria, que no coincide con la de ollo de can de Niño Ricoi (1994). La especie frankenia lavéis recibe en el diccionario la entrada franquenia, sin embargo nuestras obras de botánica la denominan queiruga mariña.

En muchas otras ocasiones podemos comprobar que los nombres que reciben determinadas plantas en el diccionario, no se ven reflejados en las obras de botánica mencionadas. Así ocurre, por ejemplo, con la astrantia major, que se recoge con la entrada astrantia en Cuveiro, mientras que en Niño Ricoi (1994) no se indica nombre vulgar alguno. Tampoco se halla la denominación vulgar de astrocarpus minor (astrocarpo, en Cuveiro), alchimilla arvensis (alquimilla, en Cuveiro), arnoseris pusilla (arnoseira, en Cuveiro), etc. En estos casos vuelve a aparecer el interrogante de la procedencia de estos nombres, ya que, como en los casos anteriores, las fuentes consultadas por Cuveiro no indican ningún nombre vulgar, ya sea gallego o castellano, y, sin embargo, salta a la vista la similitud léxica entre la entrada y la denominación latina de cada especie, lo que puede apuntar a una "adaptación" por parte de $\mathrm{Cu}$ veiro.

4 Damos por hecho que Cuveiro desconocía esta denominación, ya que no aparece en las fuentes consultadas por el autor. 
Incluso podemos observar la ausencia de algunas especies en Niño Ricoi (1994), que, sin embargo, aparecen en el diccionario. Es el caso, por ejemplo, de las plantas reseda luteolo (galda), cistus tuberaria (tuberaria), vesicastrum subterraneum (vesicastro), etc..

Desde luego, también existen alguna concordancias entre los nombres de flores y plantas que se incluyen en el diccionario y las que figuran en el Catálogo. Así recibe, por ejemplo, la planta erodium moschatum tanto en el diccionario como en el libro de botánica el nombre de almiscleiro, la especie phalaris canariensis el de alpiste, potentilla reptans el de cinco en rama y anarrhinum bellidifolium el de anarrino, etc.

\section{CONCLUSIÓN}

El diccionario de Juan Cuveiro Piñol se limita a resumir toda la información respecto a la flora gallega que en aquel momento estaba a su alcance, extrayéndola de trabajos muy reconocidos que habían supuesto un notable avance en el conocimiento de la naturaleza gallega. El gran esfuerzo de Cuveiro se halla en la recopilación y consulta del material de la época para incluirlo en su diccionario, sobre todo teniendo en cuenta que carece, como todos los autores de diccionarios del siglo XIX, de una formación lexicográfica. Muchas fuentes afirman que lo que se pretendía en aquellos tiempos era engrosar el volumen de palabras en los diccionarios, y, en nuestra opinión, este fin siempre nace y va acompañado de una sensibilidad de lo autóctono, sin que una cosa excluya la otra. El hecho de lematizar en un diccionario gallego términos castellanos demuestra el afán de totalitarismo que reinaba en aquella época de incluir todas las palabras que denoten algo gallego o que tengan relación con Galicia, aunque no exista, o se desconozca una denominación en el propio idioma. No debemos olvidar que el Rexurdimento gallego nació de un movimiento intelectual que tenía como único objetivo la preservación del gallego, pero sin proyección hacia el futuro ${ }^{5}$; el propio hecho de que las definiciones del diccionario de $\mathrm{Cu}-$

5 Véase también U. Esser (1990). 
veiro estén redactadas en castellano da buena fe de la situación lingüística de aquella época.

\section{BIBLIOGRAFÍA}

Colmeiro y Penide, Miguel (1850): Recuerdos botánicos de Galicia, Santiago de Compostela, Imprenta de la Viuda de Compañel e hijos.

Cuveiro PIÑOL, Juan (1876): Diccionario gallego, Barcelona, Establecimiento tipográfico de N. Ramírez y $\mathrm{C}^{\mathrm{a}}$.

Merino, Baltasar (1905/06): Flora descriptiva e ilustrada de Galicia, Santiago de Compostela, Tip. Galaica.

NiÑo RicoI, E. et alii (1994): Catálogo da flora vascular galega, Santiago, Xunta de Galicia-Consellería de Agricultura, Gandería y Montes.

Planellas GiRalt, José (1852): Ensayo de una flora fanerogámica gallega, Santiago de Compostela, Imprenta D. Juan Rey Romero.

QUER, Joseph (1762): Flora española o Historia de las plantas que se crían en España, Madrid, Joachin Ibarra..

RoDRíGUEZ, Francisco Javier (1863): Diccionario gallego-castellano, La Coruña, Imprenta del Hospicio Provincial.

SAmmartín, Luís A. y Helena Lago CANZobre (1998): Guía da flora do litoral galego, Vigo, Edicións Xerais.

SARMiento, Martín (1986): Catálogo de voces vulgares y en especial de voces gallegas de diferentes vegetables. Ed. José Luis Pensado, Salamanca, Universidad de Salamanca.

SeCo, Manuel (1987): Estudios de lexicografía española, Madrid, Paraninfo.

EsSER, Ursula (1990): Die Entwicklung des Galizischen zur modernen Kultursprache. Eine Fallstudie zur aktuellen Sprachplanung, Bonn, Romanistischer Verlag. 